\title{
Does the use of a colour coded inpatient capillary blood glucose monitoring chart influence outcomes?
}

\author{
DHIRAJ SHARMA ${ }^{1}$, MATTHEW GERLACH², VIVEK SHARMA ${ }^{3}$, KETAN K DHATARIYA4,5
}

\begin{abstract}
Background: Dysglycaemia in hospital inpatients is associated with harm. However, bedside capillary glucose monitoring often occurs with inadequate frequency, with inappropriate action taken when dysglycaemia occurs.

Aims: To assess whether the introduction of a colour coded blood glucose monitoring chart had an impact on glycaemic control, documentation of action taken, length of stay and mortality in an unselected cohort of hospital inpatients.

Methods: A cross-sectional study before (2014) and after (2017) the introduction of the new chart was undertaken. Data were collected for the $\mathbf{2 4}$ hours prior to inclusion in the study.

Results: Data from 56 individuals were collected in 2014 and from 161 in $2017.57 \%$ and $22 \%$ experienced dysglycaemia in 2014 and 2017, respectively. Action was taken in $\mathbf{2 4 . 2} \%$ of cases in $\mathbf{2 0 1 4}$ and in $\mathbf{5 6 . 8 \%}$ in 2017. Median length of stay was 16.1 days (IQR 8.4-39.4) in 2014 and 13.1 days (5.7-25.3) in 2017. For those who experienced dysglycaemia and in whom action was not recorded, mortality was $30 \%$ and $20 \%$ in 2014 and 2017 compared with $6 \%$ and $15 \%$, respectively, for those who had no dysglycaemia.

Conclusions: The introduction of a colour coded blood glucose monitoring chart led to more action being recorded when dysglycaemia occurred and reduced length of hospital stay and mortality.

Br J Diabetes 2019;19:19-24
\end{abstract}

Key words: diabetes, inpatient, glucose chart, outcomes

Brighton and Sussex Medical School, Brighton, East Sussex, UK Department of Medicine, Norfolk and Norwich University Hospitals NHS Foundation Trust, Colney Lane, Norwich, Norfolk, UK NR4 7UY

Cambridge University Medical School, Cambridge, UK

Elsie Bertram Diabetes Centre, Norfolk and Norwich University Hospitals NHS Foundation Trust, Colney Lane, Norwich, Norfolk, UK NR4 7UY Norwich Medicine School, University of East Anglia, Norwich, Norfolk, UK

Address for correspondence: Ketan K Dhatariya Elsie Bertram Diabetes Centre, Norfolk and Norwich University Hospital NHS Foundation Trust, Colney Lane, Norwich, Norfolk NR4 7UY, UK E-mail: ketan.dhatariya@nnuh.nhs.uk

https://doi.org/10.15277/bjd.2019.210
Introduction

Substantial data exist to suggest that dysglycaemia is associated with adverse clinical outcomes in medical and surgical inpatients. ${ }^{1-7}$ Previous authors have suggested that maintaining good glycaemic control in non-critically ill hospital inpatients reduces length of stay, mortality and short-term readmission rates. 8,9

Despite the frequency of dysglycaemia in inpatients and the evidence emphasising the importance of maintaining glycaemic control, action to treat dysglycaemia is frequently undocumented. ${ }^{10}$ What remains uncertain, however, is what interventions can be put into place quickly and cheaply to improve clinical care for this large group of potentially vulnerable inpatients.

The aim of this study was to assess whether the introduction of a colour coded capillary blood glucose (CBG) monitoring chart for bedside use had an impact on inpatient glycaemic control in an unselected cohort of hospital inpatients. In addition, we wanted to see if the introduction of the new chart was associated with any changes in the documentation of action taken, length of hospital stay and mortality.

\section{Methods}

We performed a cross-sectional study 1 year before and 2 years after the introduction of the new colour coded chart during the summer of 2015 across a large teaching hospital in East Anglia, UK. Data were collected for people on medical wards over a single 24-hour period documenting the number of dysglycaemic episodes, what action was taken and whether this action was documented in the medical notes and if it was appropriate. Dysglycaemia was defined as glucose $<4.0 \mathrm{mmol} / \mathrm{L}$ or $>15.0$ $\mathrm{mmol} / \mathrm{L}$.

Inclusion criteria included hospital inpatients with a previously documented diagnosis of type 1 or 2 diabetes mellitus who were over the age of 18 years and on a medical ward. Exclusion criteria included people undergoing surgery, those who were pregnant or post-partum, and those diagnosed with diabetes mellitus during their current admission. Data were collected from 56 individuals in September 2014 and from 160 in August 2017.

For the 20 or more years prior to the introduction of the coloured chart, our hospital used a grey-scale chart with room for plotting $C B G$, ketones and action taken (Figure 1). The new glucose chart was designed after an extensive consultation process within our hospital. The chart used a colour coded 
Figure 1. The grey scale chart used for more than 20 years prior to the introduction of the coloured chart
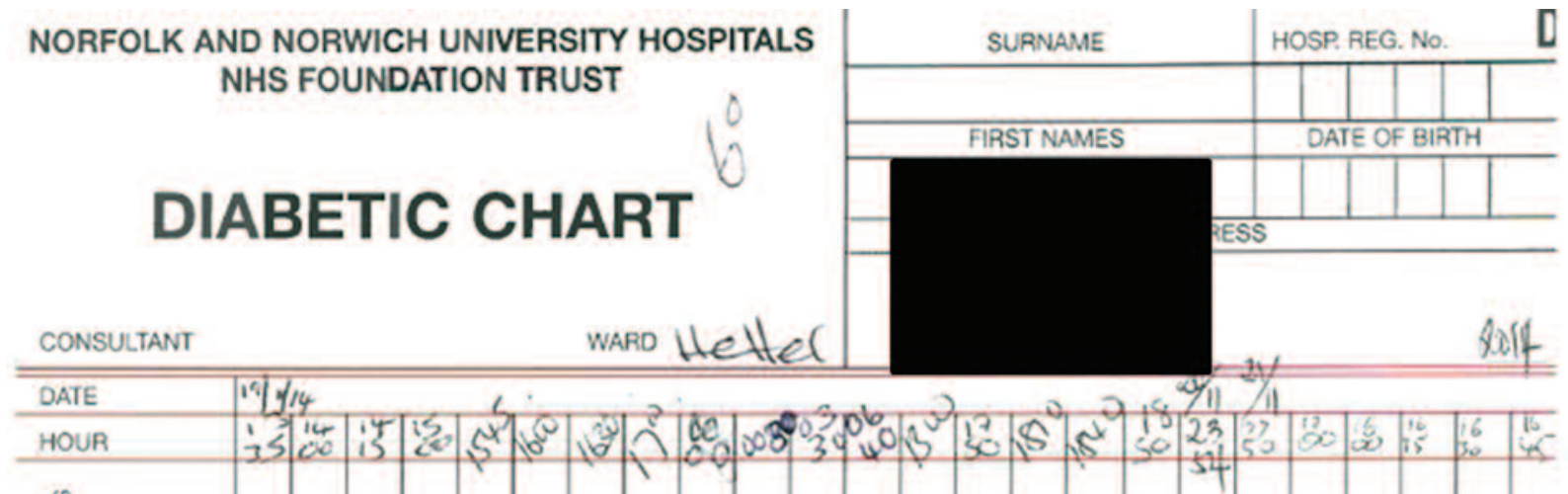

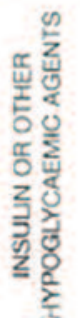
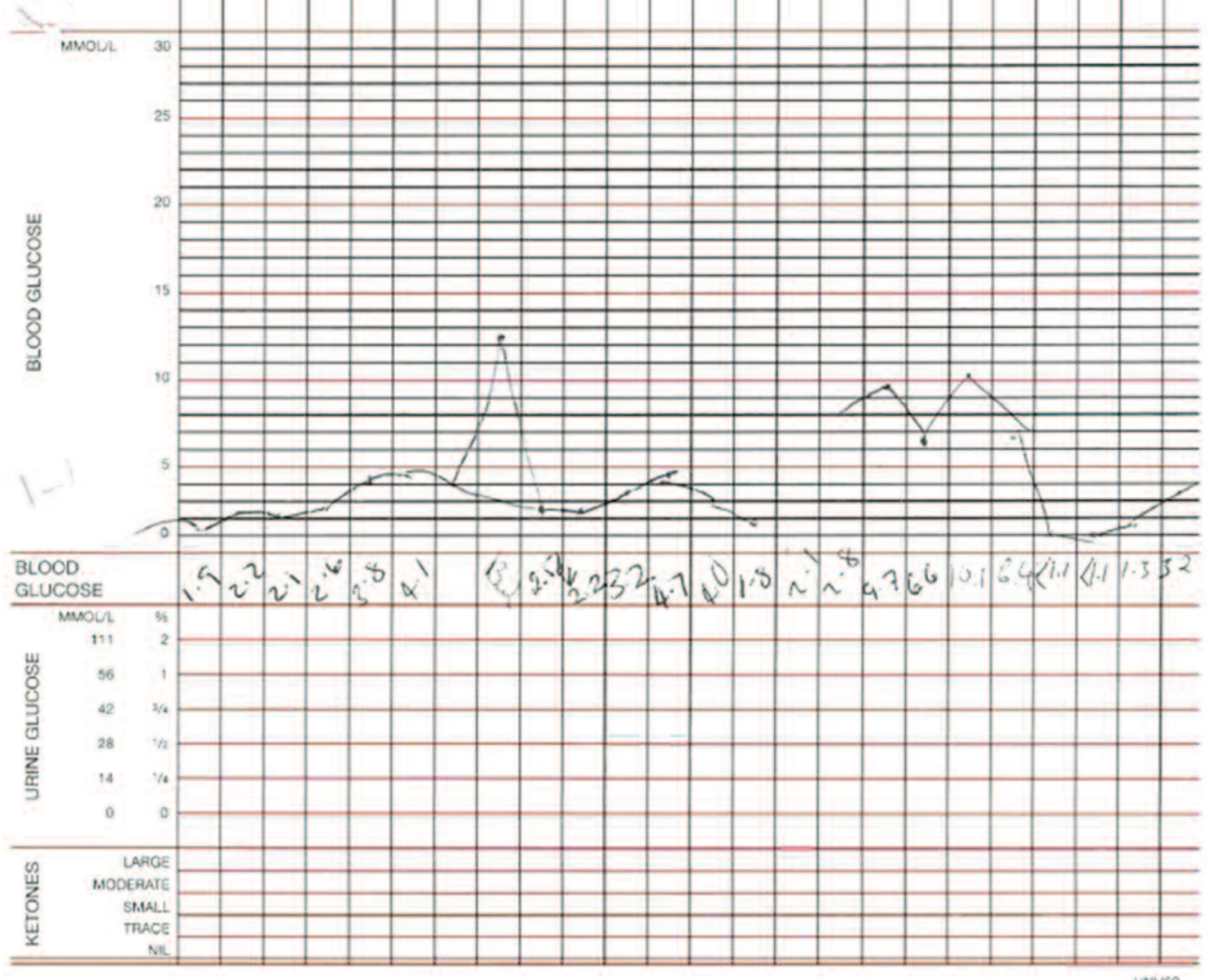
Figure 2. The new coloured blood glucose monitoring chart

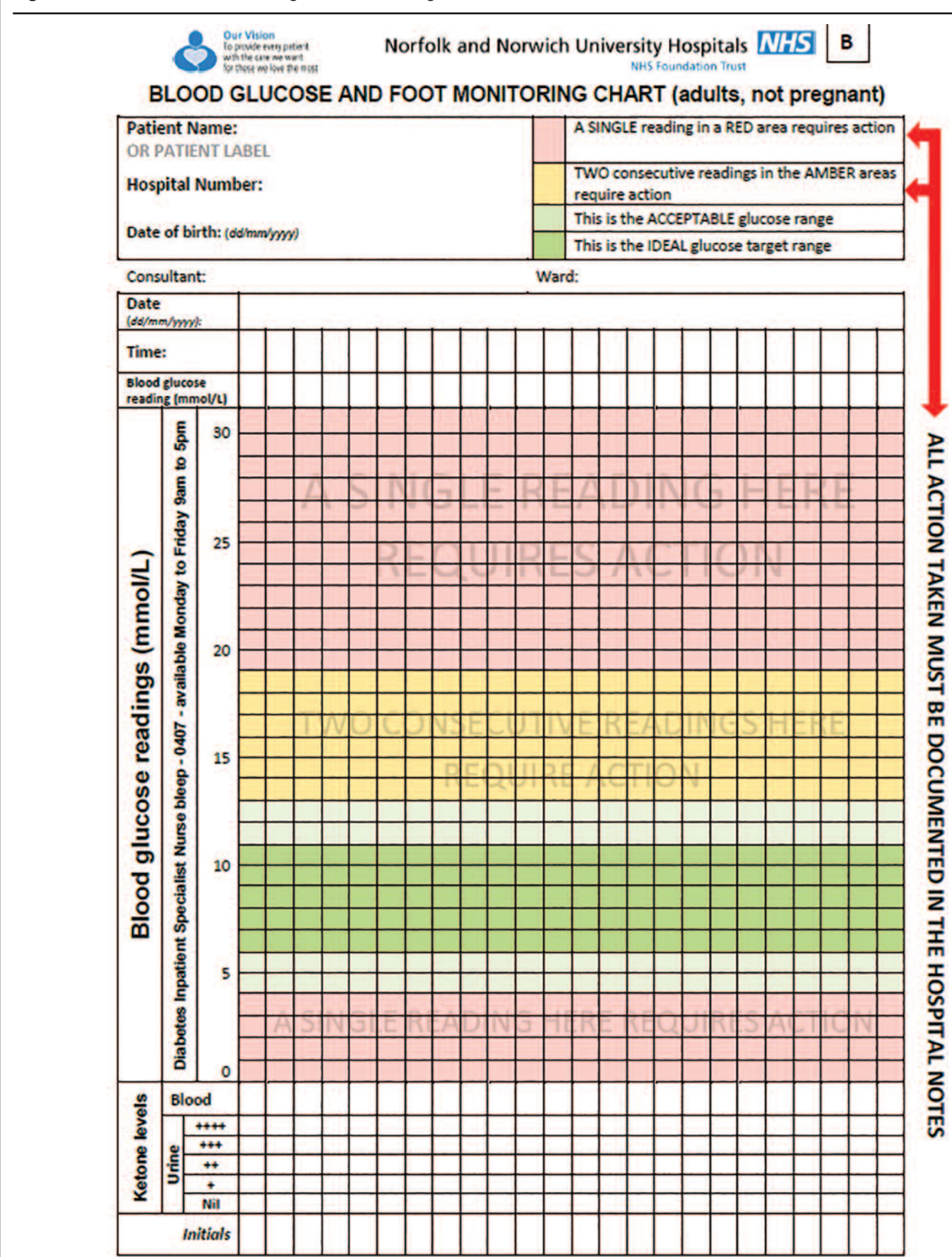


'traffic light' system. Each colour had set actions when CBG concentrations were plotted within each coloured area (Figure 2). Each action was in accordance with the Joint British Diabetes Societies for Inpatient Care guidelines. ${ }^{11}$

After collecting data from the hospital notes on dysglycaemic episodes, we later assessed the length of stay and mortality of our cohort. This was collected using the Integrated Client Environment System (Sunquest Information Systems, Uxbridge, UK).

A p value $<0.05$ was significant and results were given with $95 \%$ confidence intervals. The mean length of stay was compared using the samples t-test to look for statistical significance.

\section{Results}

Data from 56 and 161 individuals were collected in 2014 and 2017, respectively. The 2014 population included $57.2 \%$ males $(n=32)$, with an average age of 74.9 years (range 36-93 years). The 2017 population included $60.0 \%$ males $(n=96)$ with the average age being 74.1 years (range 19-112 years). In the 2014 cohort there were 16 people with type 2 diabetes mellitus (28.6\%) on diet/metformin only; in 2017 this was 60 (37.3\%).

The introduction of the colour coded chart resulted in a statistically significant reduction in dysglycaemic episodes, falling from $57 \%(n=32)$ in 2014 to $22 \%(n=36)$ in $2017(p \leq 0.0001)$. Furthermore, since the introduction of the colour charts there was also a statistically significant improvement in the recording of 'action taken to remedy the change in blood glucose', which was $24.2 \%(n=8)$ in 2014 and $56.8 \%(n=21)$ in $2017(p<0.01)$. There was also an improvement in whether actions taken were appropriate according to hospital guidelines, increasing from $24 \%(n=8)$ in 2014 to $49 \%(n=18)$ in $2017(p<0.05)$. However, this was offset by the statistically significant reduction in the recording of episodes in the notes, falling from 100\% in 2014 $(n=37)$ to $45.9 \%(n=17)$ in $2017(p<0.0001)$.

Following the introduction of the colour coded charts, the average length of stay reduced by 3 days but, because of the small numbers analysed, this reduction was not statistically significant ( $p=0.4662)$.

For those who experienced dysglycaemia and in whom no action was recorded, in-hospital mortality was 30\% in 2014 compared with $6 \%$ for those who had no dysglycaemia. This fell to $20 \%$ and $15 \%$ in 2017.

There was a significant reduction in the inappropriate use of short acting insulin to correct episodes of hyperglycaemia from $17.9 \%(n=10)$ in 2014 to $6.8 \%(n=11)$ in $2017(p=0.0321)$. The use of variable rate intravenous insulin infusions (VRIII) also fell significantly from $8.9 \%(n=5)$ in 2014 to $1.2 \%(n=2)$ in 2017 $(p=0.0134)$.

\section{Discussion}

Our data have shown that the use of a colour coded bedside CBG chart is associated with reductions in dysglycaemic episodes, hospital length of stay, inappropriate use of correct doses of bolus insulin or VRIII and mortality. In addition, there was an increase in recording in the hospital notes the action taken to remedy the change in blood glucose. These positive findings were offset by a reduction in recording of episodes of dysglycaemia in the notes.

The most recent data from the National Diabetes Inpatient Audit (NaDIA) suggest that, on average, $18 \%$ of hospital inpatients in England and Wales have diabetes. ${ }^{10}$ Previous work has shown that inpatients frequently experience high and low glucose concentrations whilst in hospital. Bersoux et al analysed glucometer data from 2.6 million inpatients and found that the prevalence of hyperglycaemic and hypoglycaemic events was $32.3 \%$ and $6.1 \%$, respectively, of those not on intensive care. ${ }^{12}$ Previous work has shown that individuals who have episodes of dysglycaemia experience more harm than those whose blood glucose concentrations remain within the reference range. $6,8,13-18$ The potential impact of these episodes goes well beyond the inpatient service, but extends to service provision across the whole hospital and into the community. ${ }^{19}$

The data from NaDIA also suggest that diabetes is often mismanaged..$^{10}$ One of the areas that NaDIA examined was the frequency of blood glucose testing, and whether it was deemed to be appropriate. The data showed that, whilst glucose monitoring is usually carried out, it is often at an inappropriate frequency with no action taken when glucose concentrations are out of range. ${ }^{10}$ This is important because guidance from the General Medical Council in their 'Good Medical Practice' document stipulates that accurate recording is an essential part of good clinical care. ${ }^{20}$

The main change made to the glucose chart was the traffic light illustration with clear instructions on when action should be taken (Figures 1 and 2). Those with dysglycaemia recorded on the charts had action taken to address this more often in 2017, and this action was deemed to be the correct action in a greater proportion of individuals. We appreciate that this could be due to a number of additional factors other than the instructions on the chart, in particular the underlying experience and knowledge among healthcare professionals - particularly because the JBDS guidelines are so extensively used. ${ }^{21}$ Although a good knowledge of diabetes management may have pre-existed, it is possible that the most junior members of healthcare professionals - for example, the Foundation Year doctors who may not have been so familiar with the JBDS documents - were guided more by the colour and instructions on the chart. However, whether this was the case remains an unanswered question.

Related to this outcome was the reduction in the inappropriate use of correction doses of short acting insulin and VRIII. This is not directly related to the new charts because they do not specify the necessary action to be taken; however, it implies that when insulin was used it was more likely to be in accordance with guidelines. The new charts instruct the person recording the blood glucose to 'take action'; this was deliberate to ensure that there was no 'proscriptive' course of action because the cause for the episode of dysglycaemia was likely to have been different. The phrase was designed to be a prompt for the individual to find out what action was necessary through discussion with a senior/specialist or by consulting the hospital guidelines.

We wanted to assess the impact of any 'action taken' on other outcomes. Average length of stay was reduced by 3 days, 


\section{Key messages}

- Dysglycaemia is a common occurrence in people with diabetes in hospital and is associated with excess harms

- The introduction of a colour coded glucose chart was associated with increased action being taken when glucose concentrations were outside of the recommended range

- Length of stay and mortality also decreased

but this was not found to be statistically significant. Once again we were unable to determine if this reduction was due to the action taken or some other factor. However, previous data have suggested that improved management of dysglycaemia has a positive impact on length of hospital stay. 6,8,9,13,16,22

For those who experienced dysglycaemia and in whom no action was recorded, mortality was 30\% and 20\% in 2014 and 2017 compared with $6 \%$ and $15 \%$, respectively, for those who had no dysglycaemia. This suggests a $33 \%$ reduction in mortality since the introduction of the coloured glucose chart. This occurred at the same time as there was a rise in the action recorded in the notes (24.2\% in 2014, and $56.8 \%$ in 2017).

We acknowledge that there are limitations to our data. There was a big difference in the number of individuals included in the study before and after the introduction of the colour coded charts. However, the numbers who experienced dysglycaemic episodes in each cohort were similar: 32 (57.1\%) in 2014 and $36(22.4 \%)$ in 2017 . We feel this makes any outcomes related to dysglycaemic episodes more comparable between the two groups. In addition, the cohorts may have had differing pathology influencing the incidence of dysglycaemia. In addition, due to the time this data collection took in 2014, we limited the cohort to medical wards only, whilst in the 2017 sample we were able to survey the whole hospital (excluding the paediatric and obstetric wards). Furthermore, other factors including ward staffing numbers and experience were not taken into account. However, the new charts may also have helped negate any variability in experience and knowledge by clearly illustrating when action should have been taken. We also acknowledge that we did not collect specific data on the development of in-hospital diabetic ketoacidosis, hyperosmolar hyperglycaemic state or severe hypoglycaemia. The most recent NaDIA data suggest that these events remain a concern, albeit occurring rarely. ${ }^{10}$ As a result, we were unable to collect data about length of stay and mortality in individuals who experienced extremes of dysglycaemia.

This study was primarily based on the documentation on the blood glucose chart and the hospital notes. Documentation has the potential to be incorrect and does not allow room for reasoning unless highly detailed. This is not usually the case. Whilst we accept that many institutions are moving towards electronic health records and electronic recording of $C B G$, there remains the necessity to clearly and accurately document what actions are taken in the event of dysglycaemia. The use of the colour coded charts appeared to have improved clinical management. It would thus be reasonable to argue that they may be a precursor to networked blood glucose monitors that are increasingly being used, allowing for rapid and automated escalations of action.

\section{Conclusion}

We have shown that, after implementation of a new colour coded bedside CBG chart, fewer individuals on medical wards experienced a dysglycaemic episode and, when they did, they were managed in better accordance with national guidelines. There was an associated non-significant but clinically meaningful reduction in length of stay in hospital. Further work needs to be done to explore the reasons for these findings and the impact of the colour coded chart on staff knowledge.

\section{Conflict of interest None. \\ Funding None.}

Author contributions DS, MG and VS collected the data and wrote the first draft of the manuscript. KD had the initial idea and wrote the final draft of the manuscript. All authors saw and approved the final submitted manuscript.

\section{References}

1. Nirantharakumar K, Marshall T, Kennedy A, Hemming K, Coleman JJ. Hypoglycaemia is associated with increased length of stay and mortality in people with diabetes who are hospitalized. Diabet Med 2012;29(12):e445e448. https://doi.org/10.1111/dme.12002

2. Turchin A, Matheny ME, Shubina M, et al. Hypoglycemia and clinical outcomes in patients with diabetes hospitalized in the general ward. Diabetes Care 2009;32(7):1153-7. https://doi.org/10.2337/dc08-2127

3. Kotagal M, Symons RG, Hirsch IB, et al. Perioperative hyperglycemia and risk of adverse events among patients with and without diabetes. Ann Surg 2016;261(1):97-103. https://doi.org/10.1097/SLA.0000000000000688

4. Frisch A, Chandra P, Smiley D, et al. Prevalence and clinical outcome of hyperglycemia in the perioperative period in noncardiac surgery. Diabetes Care 2010;33(8):1783-8. https://doi.org/10.2337/dc10-0304

5. Corsino L, Dhatariya K, Umpierrez GE. Management of diabetes and hyperglycemia in hospitalized patients. 2014. http://www.endotext.org/chapter/diabetes-treatment-strategies/management-of-the-hospitalized-diabeticpatient/

6. Baker EH, Janaway $\mathrm{CH}$, Philips BJ, et al. Hyperglycaemia is associated with poor outcomes in patients admitted to hospital with acute exacerbations of chronic obstructive pulmonary disease. Thorax 2006;61(4):284-9. https://doi.org/10.1136/thx.2005.051029

7. Bruno A, Gregori D, Caropreso A, et al. Normal glucose values are associated with a lower risk of mortality in hospitalized patients. Diabetes Care 2008;31(11):2209-10. https://doi.org/10.2337/dc08-0672

8. Brodovicz KG, Mehta V, Zhang Q, et al. Association between hypoglycemia and inpatient mortality and length of hospital stay in hospitalized, insulintreated patients. Curr Med Res Opin 2013;29(2):101-7. https://doi.org/ 10.1185/03007995.2012.754744

9. Zapatero A, Gomez-Huelgas R, Gonzalez N, et al. Frequency of hypoglycemia and its impact on length of stay, mortality, and short-term readmission in patients with diabetes hospitalized in internal medicine wards. Endocr Pract 2014;20(9):870-5. https://doi.org/10.4158/EP14006.OR

10. NHS Digital. National Diabetes Inpatient Audit (NaDIA) - 2017. 2018. https://digital.nhs.uk/catalogue/PUB30248.

11. Joint British Diabetes Societies Inpatient Care Group. Guidelines for the management of inpatient diabetes. 2014. https://abcd.care/joint-british-diabetes-societies-jbds-inpatient-care-group. 
12. Bersoux S, Cook CB, Kongable GL, Shu J, Zito DR. Benchmarking glycemic control in U.S. hospitals. Endocr Pract 2014;20(9):876-83. https://doi.org/ 10.4158/EP13516.OR

13. McAlister FA, Man J, Bistritz L, Amad H, Tandon P. Diabetes and coronary artery bypass surgery. Diabetes Care 2003;26(5):1518-24.

14. Pomposelli JJ, Baxter JK, Babineau TJ, et al. Early postoperative glucose control predicts nosocomial infection rate in diabetic patients. JPEN J Parenter Enteral Nutr 1998;22(2):77-81.

15. Seaquist ER, Anderson J, Childs B, et al. Hypoglycemia and diabetes: A report of a workgroup of the American Diabetes Association and the Endocrine Society. Diabetes Care 2013;36(5):1384-95. https://doi.org/ $10.2337 / \mathrm{dc} 12-2480$

16. Khazai NB, Hamdy O. Inpatient diabetes management in the twenty-first century. Endocrinol Metab Clinics 2016;45(4):875-94. https://doi.org/ 10.1016/j.ecl.2016.06.013

17. Umpierrez GE, Hellman R, Korytkowski MT, et al. Management of hyperglycemia in hospitalized patients in non-critical care setting: an Endocrine Society clinical practice guideline. J Clin Endocrinol Metab 2012;97(1):16-
38. https://doi.org/10.1210/jc.2011-2098

18. McAlister FA, Majumdar SR, Blitz S, Rowe BH, Romney J, Marrie TJ. The relation between hyperglycemia and outcomes in 2,471 patients admitted to the hospital with community-acquired pneumonia. Diabetes Care 2005; 28(4):810-5.

19. Liebl A, Khunti K, Orozco-Beltran D, Yale JF. Health economic evaluation of type 2 diabetes mellitus: a clinical practice focused review. Clin Med Insights Endocrinol Diabetes 2015;8:13-19. https://doi.org/10.4137/CMED.S20906

20. General Medical Council. Good medical practice. 2014. https://www.gmcuk.org/-/media/documents/good-medical-practice---english-1215_pdf51527435.pdf

21. Sampson M, Jones C. Joint British Diabetes Societies for Inpatient Care: Clinical guidelines and improving inpatient diabetes care. Diabet Med 2018;35(8):988-91. https://doi.org/10.1111/dme.13672

22. Umpierrez GE, Isaacs SD, Bazargan N, et al. Hyperglycemia: an independent marker of in-hospital mortality in patients with undiagnosed diabetes. J Clin Endocrinol Metab 2002;87(3):978-82. https://doi.org/10.1210/ jcem.87.3.8341

\section{THE BRITISH JOURNAL OF Diabetes}

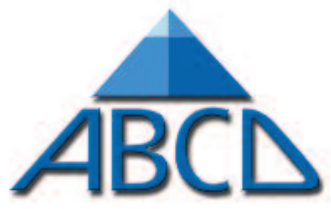

The Journal of the Association of British Clinical Diabetologists

\section{CALL FOR PAPERS}

The British Journal of Diabetes is the official journal of the $A B C D$ and primarily seeks to fulfil the needs of diabetes practitioners. Our mission statement is to provide the diabetes multi-disciplinary team with free, accessible, authoritative, peer-reviewed articles, offer a context for practice and facilitate understanding of professional challenges.

We are keen to encourage new, relevant and interesting content to help support improvements in patient care and clinical practice. We are inviting contributions of the following article types;

ORIGINAL ARTICLES / RESEARCH: any topic / area of diabetes or vascular research which is clinically meaningful to diabetes health care professionals.

REVIEW ARTICLES: we welcome reviews that look at specific topics and explore the current literature.

CURRENT TOPICS: short articles or reports on subject of present relevance in the diabetes world.

CASE REPORTS: we are keen to encourage clinical cases which elucidate interesting practice points, case scenarios, good clinical images (with patient permission) and common problems faced by diabetologists.

ACHIEVING BEST PRACTICE: do you run a good diabetes service? Articles which describe innovative approaches to patient care, cost effective management and good organisation or service re-design.

Author instructions: http://bjd-abcd.com/index.php/bjd/about/submissions\#authorGuidelines Journal website: http://bjd-abcd.com/index.php/bjd/index

Enquiries: editor1@bjd-abcd.com and ja@bjd-abcd.com

Formerly The British Journal of Diabetes \& Vascular Disease

www.bjd-abcd.com 